\title{
Mehr Todesfälle bei blutarmen Op.-Patienten
}

- Patienten, die mit einer Anämie in eine große Operation hineingehen, haben eine schlechtere Prognose: Morbidität und Mortalität nach 30 Tagen fallen deutlich höher aus als bei Patienten ohne Blutarmut.

Die negativen Auswirkungen einer Anämie bei kardiologischen Operationen sind bekannt. Dass Blutmangel bei chirurgischen Eingriffen generell einen Risikofaktor darstellt, wird nun durch eine Datenbankstudie belegt. Dazu waren aus einem prospektiven Register die Daten aller Patienten herausgezogen worden, die sich im Jahr 2008 einer nicht kardiologischen Operation unterzogen hatten.

Insgesamt wurden die Daten von 227425 Patienten ausgewertet. Bei 69229 (30,44\%) Patienten bestand präoperativ eine - in den meisten Fällen leichte - Anämie. Diese Patienten waren im Schnitt etwas älter und hatten mehr Begleiterkrankungen. Ihr schlechteres postoperative Abschneiden war daher wenig überraschend. Eine Anämie erwies sich aber auch dann als prognostisch ungünstig, wenn alle begleitenden Risikofaktoren abgeglichen wurden: Die Sterblichkeit in den ersten 30 Tagen nach der Op. lag immer noch um $42 \%$ höher als bei Patienten mit normalem Hämatokrit. Selbst eine leichte Anämie trieb die Mortalität in die Höhe (OR 1,41).

Auch die 30-Tage-Morbidität war bei den blutarmen Patienten - um 35\% - erhöht. Betroffen waren Patienten mit leichter wie mit mittelschwerer bis schwerer Anämie (OR 1,31 und 1,56). Nahezu alle Arten von Komplikationen, inkl. respiratorischer, urologischer, septischer, thromboembolischer und Wundkomplikationen, traten bei ihnen vermehrt auf. Die Autoren empfehlen, „vor einer Operation die Behandlung einer Anämie in Erwägung zu ziehen". BS • Mussallam KM et al. Lancet 2011; published online Oct 6, 2011

\section{KOMPLIKATIONEN NACH GELENKERSATZ}

\section{Vorsicht bei Adipösen: Hüft-TEP springt aus der Pfanne}

- Die 5-Jahres-Ergebnisse nach Implantation eines künstlichen Hüftgelenks sind bei adipösen Patienten schlechter als bei normalgewichtigen. Dies bestätigt eine prospektive Studie mit 1617 Patienten, denen zwischen 1998 und 2005 wegen einer Arthrose eine Hüftgelenksprothese (HTEP) eingesetzt worden war.

In den fünf Jahren nach der HTEP kam es zu 42 Luxationen, 39 Revisionen, 15 tiefen und 83 oberflächlichen Infektionen. Dabei erwies sich ein höherer BMI als unabhängiger Risikofaktor für Luxationen, oberflächliche Infektionen sowie ein schlechteres Abschneiden im Harris Hip Score (HHS) und beim allgemeinen Gesundheitszustand, gemessen mit dem SF-36.

Eine Dislokation der Prothese trat bei 6,8\% der Patienten mit einem BMl von $35 \mathrm{~kg} / \mathrm{m}^{2}$ und mehr auf, aber nur bei 1,5\% der Patienten mit einem BMl unter $25 \mathrm{~kg} / \mathrm{m}^{2}$. Nach Berücksichtigung anderer Störfaktoren er- gab sich ein linearer Anstieg des Luxationsrisikos mit dem BMI.

Oberflächliche Infektionen nahmen vor allem ab einem BMI von $35 \mathrm{~kg} / \mathrm{m}^{2}$ stark zu. Die Rate lag hier bei $14,2 \%$, bei einem BMI unter $25 \mathrm{~kg} / \mathrm{m}^{2}$ betrug sie nur $4,4 \%$.

Für den HHS wurde ein Verlust von 3 (von insgesamt 100) Punkten durch einen um zehn Einheiten höheren BMI errechnet. BS Davis AM et al. J Bone Joint Surg 2011; 93-B: 1178-82

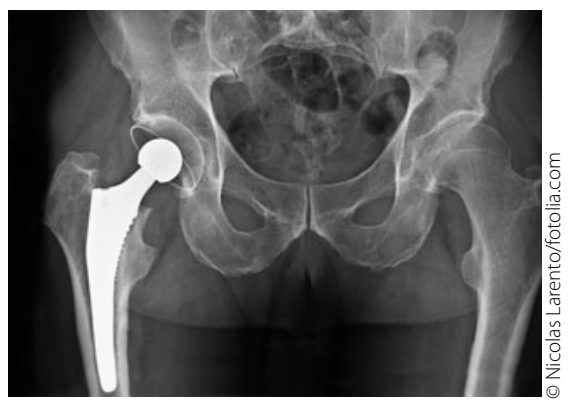

Bei Schlanken hält die Prothese besser.
SCHWANGERSCHAFTSDIABETES Oft reicht Metformin

- Gestationsdiabetes kann mit Metformin oft ebenso effektiv behandelt werden wie mit Insulin, so das Ergebnis einer neuen Metaanalyse.

Mit der sprunghaften Zunahme der Adipositas wird auch der Schwangerschaftsdiabetes immer häufiger. In Europa muss etwa bei $2-5 \%$ aller Schwangerschaften mit dieser Komplikation gerechnet werden, so Robert Fraser aus Sheffield/England.

Die Stoffwechselstörung prädestiniert für eine Reihe von Komplikationen, z. B. kindliche Makrosomie, Hypoglykämien unter der Geburt und fetaler Hyperinsulinismus. Mütter mit

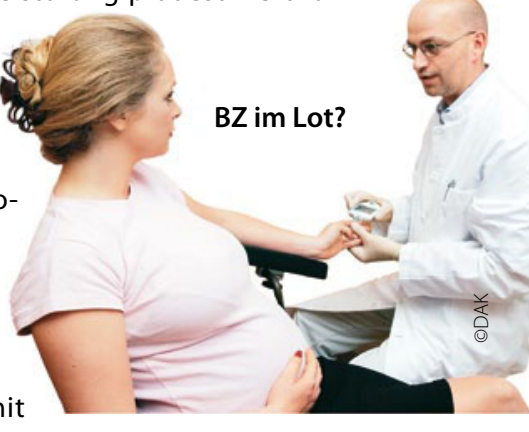
Gestationsdiabetes

entbinden häufiger per Sectio. Kinder aus solchen Schwangerschaften haben ein erhöhtes Diabetesrisiko später im Leben.

\section{Therapie verhindert Komplikationen}

Kann die Behandlung des Gestationsdiabetes perinatale Komplikationen verhindern? Dazu wurden zwei Studien durchgeführt. Eine gemeinsame Betrachtung beider Studien in einer Metaanalyse zeigt: Die Therapie mindert u. a. die Risiken für Makrosomie und Schulterdystokie.

In der Therapie des Gestationsdiabetes stehen Diät, körperliche Aktivität und Gewichtsreduktion im Vordergrund. Medikamentös werden Metformin oder Glibenclamid empfohlen, bei Frauen mit Gewichtsproblemen v. a. Metformin. Orale Antidiabetika sind einer Insulintherapie bei Gestationsdiabetes bezüglich Stoffwechselkontrolle oder Verhinderung neonataler Komplikationen durchaus ebenbürtig, so das Ergebnis einer kürzlichen Metaanalyse. Ein systematisches Screening auf Gestationsdiabetes lohnt immer dann, wenn das individuelle Risiko größer als 4,2\% ist, so Fraser.

DE

Jahreskongress der European Association for the Study of Diabetes, Lissabon, September 2011 